\title{
Development of Environmental Monitoring System with Wireless Sensor Networks
}

\author{
A. Ghobakhlou, S. Zandi, and P. Sallis

\begin{abstract}
Geoinformatics Research Centre, Auckland University of Technology, Auckland, New Zealand
\end{abstract} \\ Email: akbar@aut.ac.nz
}

\begin{abstract}
Wireless Sensor Network (WSN) technology is becoming increasingly popular, particularly as applied to a variety of monitoring and tracking applications. Recent developments and advances in both information processing and wireless sensor technologies have provided environmental management systems with capabilities of real-time remote location monitoring. WSN enables monitoring and management of a large set of environmental data including climatic, atmospheric, plant and soil parameters that influence cropland growing environments. Real-time sensor data collection is used for accurate illustrations of current conditions while forecasting future conditions and risks. The real time information from the fields can provide a solid base for farmers to adjust strategies at any time. Instead of making decisions based in some hypothetical average condition, which may not exist anywhere in the reality, a precision farming approach recognizes differences and adjusts management actions accordingly.
\end{abstract}

Modular architecture minimises the software upgrade down time and enables hardware reusability. If any firmware upgrade required, preassembled and pre-programmed microcontroller module can be sent to the end user. Recent developments and advances in wireless technology as well as affordability give rise to this emerging field in the realm of precision farming. WSN can operate in a wide range of environments and provide advantages in cost, size, power, flexibility and distributed intelligence, compared to wired ones. Recent advances in wireless sensor networking technology have led to the development of low cost, low power, multifunctional sensor nodes. Sensor nodes enable environment sensing together with data processing.

This paper describes a framework for using WSN and a dynamic web application designed to monitor changes in environmental conditions (especially microclimates) using in-field sensor data collected from vineyards. The monitoring is part of a larger proposed system including hardware and software designed to collect field data in harsh terrain conditions. A database

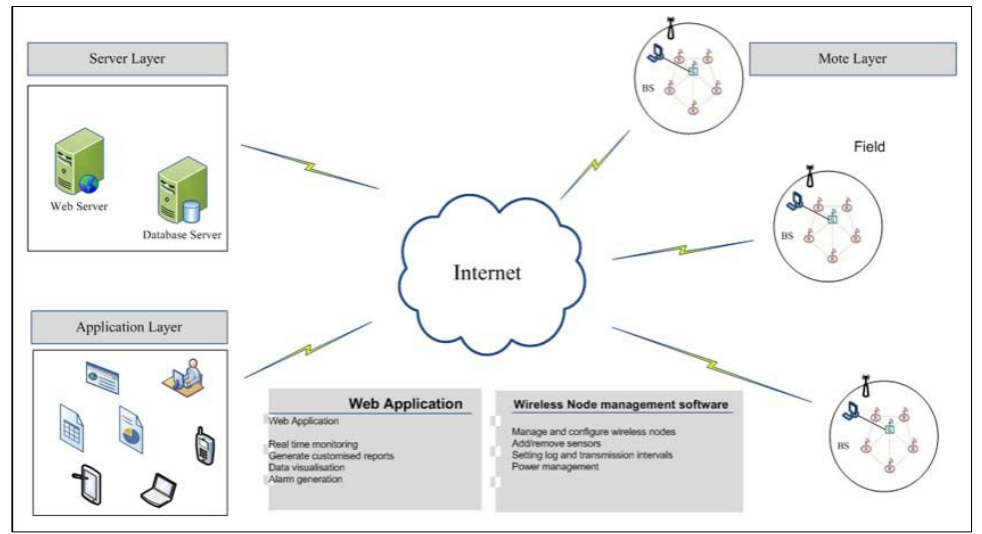

Figure 1: A schematic view of WSN architecture management system with associated software performs data analysis and visualisation of data received from various monitoring stations in five countries. It has the capability to compare and analyse data from different sensor nodes, different sensors within the same sensor node and selected sensors. Data is successfully received from the deployment sites and has the capability of real-time streaming and analysis via internet browsers.

Keywords: Wireless Sensor Networks (WSN), modular sensor node, environmental monitoring, vineyard monitoring 


\section{INTRODUCTION}

Environmental monitoring comprises the processes, actions and data collection methods used to observe the condition of the environment. Environmental data are an important input to many agricultural models, since crop yields depend on environmental conditions. To achieve a reliable and robust foundation for managing agricultural environments, there is a desperate need for data with high spatial resolution, wide thematic range and high thematic resolution (Lee et al., 2010). Information required includes but is not limited to: climate data, soil properties, crop yield, crop nutrients, water content, and pest conditions. Microclimate monitoring conducts a precise measurement, recording and storing of data of selected parameters within the deployed environment. This allows growers to closely monitor and control many aspects of crop production. Remote and local sensors or sensor networks can be applied to monitor plant nutrient and moisture needs, soil conditions, and plant's health including insect and disease detection.

Recent advances in wireless sensor technologies have led to the development of low cost, low power, compact sensor nodes. This provides enormous opportunities in research and development of numerous applications. Wireless Sensor Networks (WSN) are seen as one of the most promising contemporary technologies for bridging the physical and virtual worlds thus, enabling them to interact. WSNs are used in both military and civilian applications including wireless data acquisition, smart buildings, target tracking, habitant monitoring, environmental contaminant detections and precision agriculture (Cao et al., 2008, Camili et al., 2007).

A WSN usually has little or no infrastructure. It consists of a number of sensors nodes (few tens to thousands) working together to monitor a region to obtain data about the environment. There are two types of WSNs: structured and unstructured. Unstructured WSN contains dense collection of sensor nodes and often deployed in ad-hoc manner in the field. In an ad-hoc deployment, sensor nodes can be dropped from a plane and randomly into the target area. In structured WSN on the other hand, all or some of the sensor nodes are deployed in a pre-determined locations. Structured network has fewer nodes and can be deployed with lower network maintenance and management costs (Deborah et al., 1999).

\subsection{Agriculture and Field Monitoring}

Agricultural crop yields depend on environmental conditions, and the response of plant growth to changing environmental conditions is extremely complicated. The complexity is caused by chaos (Hirafuji and Kubota, 1994), and generally speaking, enormous amounts of time-series data are required to predict/ control such chaotic systems. Thus, there is need to collect enormous amounts of data in the field. Laurenson et al. (2002) developed a middleware named MetBroker that provides access to many different weather databases. As a result, weather data from about 23,000 sites are integrated into a single set. While this seems like a large number, in fact only one weather station exists per $21,000 \mathrm{~km}^{2}$ on average. However, to make matters worse, important data such as solar radiation, soil moisture and $\mathrm{CO}_{2}$ concentration, which affect plant growth, are not collected (Lee et al., 2010).

Thus, farmers and researchers must obtain data at their sites by themselves, but conventional weather stations are too expensive and too large. So far, data loggers have been employed for such purposes, but users must visit these stations frequently simply to collect the data. To solve these problems, sensor networks are desirable. The WSN ability to simultaneously capture and relay real time data for analysing the variability in climate change and its effects on plant physiology is significant for different crop productions. This is because modelling the relationships between climate variability requires both data on the cause and effects recorded without any time discrepancies. Complexity in the models increases with spatial information combined with other environment related parametric variables. Gaining more insights into natural systems and their functioning including climate change involves many complex dynamic and diverse processes with nonlinear interactions that pose huge challenges to modellers (Suri et al., 2006).

The work presented herein is an updated and extended version of a paper in (Ghobakhlou et al., 2010) which is realisation of a concept first outlined in what was called the Eno-Humanas Project (Sallis et al., 2008). It describes a system for gathering (sensing), monitoring and analysing climate, and atmosphere, plant and soil data. It is specifically designed for microclimate analysis in agro-metrological environments such as vineyards. Modelling the effects of macro-micro climate changes has begun in this project with the use of WSN data obtained from vineyards located in three continents. This enables observations of the variability in global climate change across the continents using prediction model values provided by NASA and other institutions. The models and results will be used in a comparative analysis on climate change effects on viticulture, especially its variability and its influence on grapevine "cultivars" or varieties and wine quality, such as aroma, 
colour and mouth feel as climate change effects on viticulture is described to be dramatic and varying across the globe significantly.

Deployment of WSN for microclimate monitoring in vineyards and orchards provides a decision support system tool for growers to:

- Identify the amount of nutrients deficiency within the targeted crop fields.

- Conserve water through data monitoring soil moisture levels to determine when and where there is lack of water in the root system, despite the moist appearance of the ground surface.

- Reduce potential profit loss by providing timely and accurate data that could be used for predicting undesirable climate changes and triggering quick action to protect fragile crops.

- Control fungal diseases such as powdery mildew. The grapevine powdery mildew control programs have been more effective when fungicides were applied as protectants. Online monitoring of temperature and leaf wetness assists growers to predict the occurrence of infection and decide what fungicide program should be performed in terms of product and application interval.

- Increase productivity and minimise labour costs by performing timely thinning, pruning, spraying, and harvesting.

- Enable on-demand access to real-time information, environmental data is sent from the field to the Internet for viewing anytime, from anywhere in the world with a PC or Internet-enabled mobile phone.

A wireless network such as the one discussed herein will enable the vineyard management to decide on the kind of measures (i.e., sprinkler system, gas/ turbine heaters/ helicopter and a schedule) required for example, to prevent frost damage to the crops. Further details on the frost prediction and wireless sensor network issues could be found in (Sallis et al., 2009). Some of the challenges and considerations on the use of these sensors and technologies for specialty crop production are also discussed.

\section{WIRELESS TECHNOLOGY}

WSN is one of the most significant technologies in decades eliminating connectors, provides safe/flexible connectivity, it improves resources sharing, easy installation and mobility. The other advantage is that these systems require a low micro power levels, thus it can last for longer period of time (Jose and Gutierrez, 2005). Two major protocols are used in wireless networking, IEE 802.15.4 and ZigBee stack. The ZigBee network stack sits on top of IEEE 802.15.4 standard Medium Access Control (MAC) and Physical (PHY) layers. MAC and PHY layers define the RF and communication components between other devices. ZigBee stack contains the network layer, an application layer and security service provider (SSP) (Egan, 2005). These protocols have their own limitations and advantages. The main limitation for both protocols is low data rate (narrower bandwidth). Therefore these protocols are suitable for low data transmission applications. As the data rate is increased the power consumption, cost and complexity of the system increases geometrically. Since sensor nodes are often located in remote locations, power consumption needs to be minimal, preferably battery operation for end of product life. Moreover sensor data does not require wide band-width and therefore ZigBee protocol is suitable for small sensor networks.

\subsection{Wireless Network Topologies}

Three main network topologies used in wireless net-working are point-to-point, star and mesh. Each of these topologies has their own advantages and disadvantages in different applications. Their main difference is the use and behaviour of network components.

Mesh topology is also sometimes called a peer-to-peer system. This system is an ad hoc multi-hop system. Mesh networks are based on the ZigBee protocol, which means each node can be used to send and receive data. In this manner, network consists of multiple redundant communication paths, which can be used in an event of node failure. Therefore, data will reach its destination reliably via the intermediate nodes. The mesh network has three important properties: Self-Configuring, Self-Healing and Scalability. A ZigBee mesh network configures itself automatically. The network identifies new nodes and automatically includes them in the network. Moreover, if one node fails the network re-routes the message through an alternative path. According to the ZibBee mesh network standards, it can support up to 65,536 network nodes (clients) (Egan, 2005). 


\subsection{Challenges}

Power consumption remains a critical operational constraint and the central design consideration for WSNs, whether they are battery powered or energy harvesters. The sensor node lifetime typically exhibits a strong dependency on battery life. In many cases, the wireless sensor node has a limited power source, and replenishment of power may be limited or impossible altogether. The power management and power conservation are critical functions for sensor networks, and one needs to design power-aware protocols and algorithms (Sohraby et al., 2007). Two approaches to reduce the power consumption of WSN in (Srivastava, 2010) are:

Ultra Low-Power Networks: Minimizing current consumption is only part of the solution. Several essential issues are key to developing a low-power wireless sensor application i.e. efficiently harvesting, converting, and storing the energy as well as using available energy in the most efficient way, without compromising performance (range, data rate, latency, and/or standards compliance). This is becoming possible with development of the ultra-low-power transceiver radio chip. By letting the microprocessor sleep until it is needed, it is possible to save over $65 \%$ of energy usage as compared to the typical always-on transceiver.

Energy Harvesting: Energy harvesting devices can usually be divided into two general categories namely "burster" and "tricklers". A burster produces a short but strong peak of energy and examples are dynamo or micro-generator. Whereas, the trickler generally behaves as an energy source with a faint but constant power. The most famous trickler energy is the solar cell. Some varieties of solar cells are capable of extracting energy from limited indoors light. Solar cells are the only energy harvesters that have already been produced in high enough quantities and with sufficiently low cost for use in WSNs. However, small rechargeable batteries are still needed to store power for times when the WSNs need to be operating while there is no light present. This presents a need for human intervention, which diminishes the automated character of these solutions.

\section{SYSTEM ARCHITECTURE}

The proposed system architecture (Ghobakhlou et al., 2010) consists of sensor nodes located in critical locations within a crop field (vineyard in this case) for collecting weather, atmospheric and environmental data as well as plant related data such as leaf wetness and sap flow. Figure 1 shows a schematic view of system architecture consisting of three layers namely, mote, server and application layers.

Mote layer: This layer consists of all the wireless sensor nodes and a Base Station (BS). Each node has one or more sensors plugged into the hardware device with a transmitter, power supply and microcontroller. The nodes are distributed over an area of interest uniquely arranged in a manner that the distance between the nodes does not exceed the maximum RF communication range. Therefore, energy optimized routing becomes essential. Data transmission from sensor nodes to the BS depends on application maybe continuous, event driven, query-driven or hybrid. In the continuous approach, data is transmitted to the BS periodically according to predetermined intervals. In query and event driven models, data is transmitted when an event takes place or a query is generated from the BS. The Hybrid model uses combinations of these approaches to transmit data from sensor nodes to the BS. Various types of routing protocols such as data-centric, hierarchical and locationbased protocols are available (Garcia, 2006).

Server layer: Data are sent to the data server from the BS through the internet. Two main tasks performed by data server are to:

1) Obtain and process data from the BS.

2) Populate database with WSN data and enabling the application layer to access WSN data.

The server layer also deals with on-time data delivery from the BS and generates alarm when an undesirable event takes place.

Application layer: This layer allows users of the system to have remote access to WSN data using web browsers. This provides a powerful tool to visualize real-time WSN data and compare data from various nodes. In addition, the BS can be accessed remotely to modify sensor nodes' configurations.

\subsection{Modular Sensor Architecture}

During the past few years modular sensor node architecture is shown to be popular due to increasing demand on more energy efficient micro processors, accurate and sensitive sensor developments, improved wireless radios and improvement of wireless software architecture for efficient data management (Portilla et al., 2006). 
Modularity design imposes greater flexibility for the end product. Same node can be utilised for specific application equipped with required sensor modules. This is highly desirable when each sensor node collecting microclimate, atmospheric and plant data within different vineyards requires different sets of sensors. Figure 2 illustrates the building blocks of the proposed modular sensor design. The wireless microcontroller module controls the whole data communication aspect, while sensor data coordinating microcontroller module, collects and calibrates the data. It is possible to introduce various types of sensor modules required by an application using this technology.

\subsection{Modular Design}

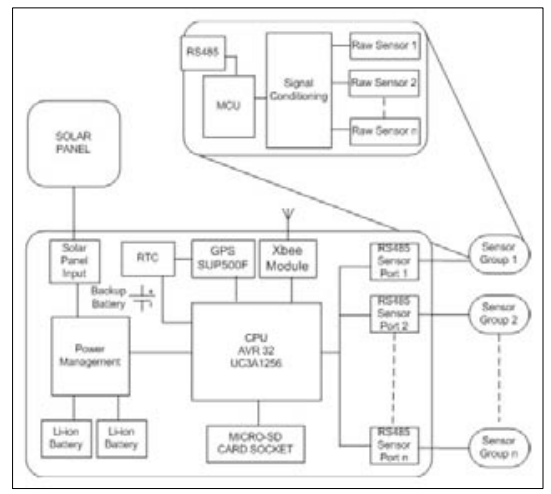

This design allows for including up to 16 different sensors (see Table 1) and two controller modules. Related sensors are grouped together (e.g. temperature and humidity) while others such as leaf wetness is on its own

Table 1: List of sensors group and sensor type

\begin{tabular}{|c|l|}
\hline Sensor Group & \multicolumn{1}{|c|}{ Sensor Type } \\
\hline \multirow{4}{*}{ CLIMATE } & Air Temperature \\
\cline { 2 - 2 } & Air Pressure \\
\cline { 2 - 2 } & Humidity \\
\cline { 2 - 2 } & Wind Speed \\
\cline { 2 - 2 } & Wind Direction \\
\cline { 2 - 2 } & Rainfall \\
\hline \multirow{2}{*}{ SOIL } & Soil Temperature \\
\cline { 2 - 2 } & Soil Moisture \\
\hline \multirow{4}{*}{ PAST } & Leaf Wetness \\
\cline { 2 - 2 } & Sap Rise \\
\hline \multirow{2}{*}{ Radiation } & Carbon Monoxide $(\mathrm{CO})$ \\
\cline { 2 - 2 } & Carbon Dioxide $\left(\mathrm{CO}_{2}\right)$ \\
\cline { 2 - 2 } & Ozone $\left(\mathrm{O}_{3}\right)$ \\
\cline { 2 - 2 } & Methane $\left(\mathrm{CH}_{4}\right)$ \\
\cline { 2 - 2 } & UV Radiation \\
\hline & Solar Radiation \\
\hline
\end{tabular}
and externally exposed pluggable modules. There are two levels of

controller modules, board level controller and communication level controller. ATmega1281 microcontroller is used for board level communication. This powerful microcontroller consists of 16 Analogue to Digital Conversion (ADC) channels and 4 UART ports. This microcontroller can operate up to $16 \mathrm{MHz}$ at $5 \mathrm{VDC}$.

The main aim of this board level controller unit is to maintain the interfacing between different sensor modules and transfer the processed data to the communication level controller module via RS232 communication port. The power management of the sensor modules is monitored by ATmega1281 microcontroller. The power management is archived by shutting down all the sensors when they are not in service. The service time will be defined by either pre allocated time interval or request from the main coordinator unit. When the units are run on preallocated mode, microcontroller extracts the reading from the sensor module in defined intervals. The second mode is interactive process between coordinator and router nodes. The coordinator nodes can request data from each individual router node, in any random order according to the user inputs. The main purpose of the communication level controller is to maintain the XBee mesh network and transmit the data to the coordinator node. The Taxes Instrument CC2431 wireless microcontroller is used to create the mesh network. The main advantage of this microcontroller is that it has built-in radio transceiver, which operates at free commercial $2.4 \mathrm{GHz}$ frequency band.

Once data is received by the BS' Xbee RF transceiver, it will be transferred to the computer via USB interface. The node may hop the data to the BS via adjacent nodes. Each node will have a list of neighbouring node ID table. If for any reason hopping through a neighbouring node fails, node will go through the list of IDs to send the data to the next closest node.

Modular design minimises the software upgrade down time. If any firmware upgrade required, preassembled and pre-programmed microcontroller module can be sent to the end user. Then user can replace the preprogrammed module with the existing module. This module can be sent back to the workshop for firmware upgrade. More powerful controller module maybe introduced, with minimum hardware changes.

\section{IMPLEMENTATIONS}

To achieve objectives described in Eno-Humanas project (Sallis, 2008), a WSN prototype was designed and developed specifically for gathering and monitoring environmental data within vineyards. Both hardware and software were designed and built by researchers at Auckland University Technology (AUT).

\subsection{Hardware design}

Both sensor node and base station as depicted in Figure 4 are using wireless plug-in module. The plug-in board is based on CC2431 wireless micro controller. This microcontroller has on chip $2.4 \mathrm{GHz}$ wireless Xbee RF, $128 \mathrm{~KB}$ in-system programmable flash and hardware based location awareness. This allows addressable 
communications between nodes. Data may be sent to individual nodes (point-to-point), or to all nodes in range (point-to-multipoint) using a broadcast address. Sensor nodes used are small low-cost with two microcontrollers which have their own built-in micro-processor. These nodes can automatically set up an adhoc wireless communication network with other motes that are within the RF range. Nodes are battery powered allowing wireless communication capabilities and can be supported by power solar cells. Solar energy is stored in rechargeable (secondary) batteries (see Figure 3). This is essential in this application where maintenance operations such as battery changing are impractical. Sensors may be configured to send data to the BS in various manners.

In this study, data are sent to the BS periodically at varies intervals for different sensors. There In addition, an ultra light minicomputer with wireless communication capability is used to receive sensed data from sensor nodes. The BS also can be accessed remotely via internet to upload sensor data to the main off-site server. Figure 3 is showing a screenshot of the Sensor Node Management (SENOMA) GUI software designed for monitoring and configuring sensors attached to each node (e.g. interval time for each sensor), data logging and server upload.

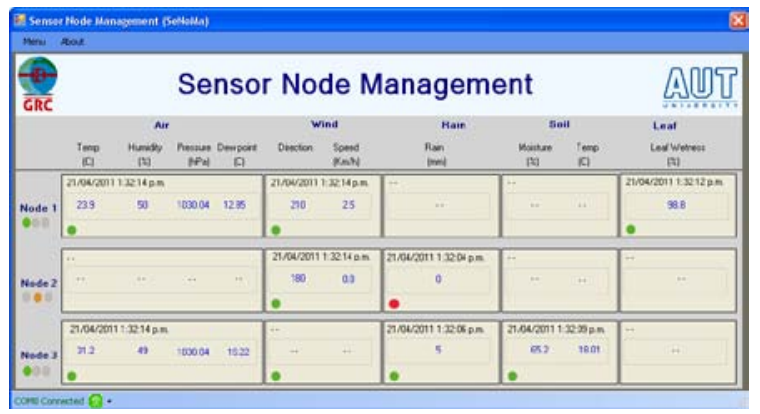

Figure 3: Screenshot of sensor node management GUI

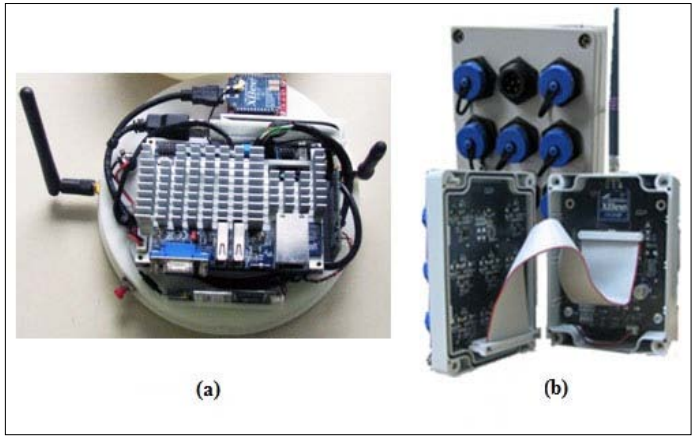

Figure 4: (a) Base station (b) Modular sensor node

\section{Sensor node}

The sensor node (Figure 4.b) can be divided into two main modules; sensor module and wireless module. The CC2431 controller module collects the sensor readings via Analogue to Digital Converters (ADC) and transmits the data to the BS (Figure 4.a). The sensor unit is powered via 2 X 2000mAh Li-Ion rechargeable batteries. Due to limitation on flash memory, raw data is sent to the BS without any processing. The $\mathrm{BS}$ converts ADC reading into appropriate values by using conversion factors. The sensor modules can accommodate up to sixteen different environmental sensors as listed in Table 1. Figure 3 shows a screenshot of the main SENOMA GUI that monitors WSNs connectivity. Sensor modules can be added/removed as required.

\section{WEB MONITORING}

A web application was developed to display the sensor data enabling users to interactively access the WSN data. Sensor data are uploaded from each BS to a remote central server. The server side process the incoming data and populates the database. It allows live monitoring and visualization of climate, atmosphere, plants and soil data from each vineyard. It also provides a dashboard for displaying sensor readings and derived parameters such ad dew point (refer to Figure 5). Historical data displayed in grid and graphical formats which can be customized to provide better visualisation of logged data. Figure 5 shows web monitoring application displaying the Artisan vineyard's environmental data.

\section{DISCUSSION}

Generally speaking most of commercial wireless sensor node architectures follow a similar design involving a single microcontroller and a number of connected components. Alternative architectures for wireless sensor nodes such as modular architecture proposed

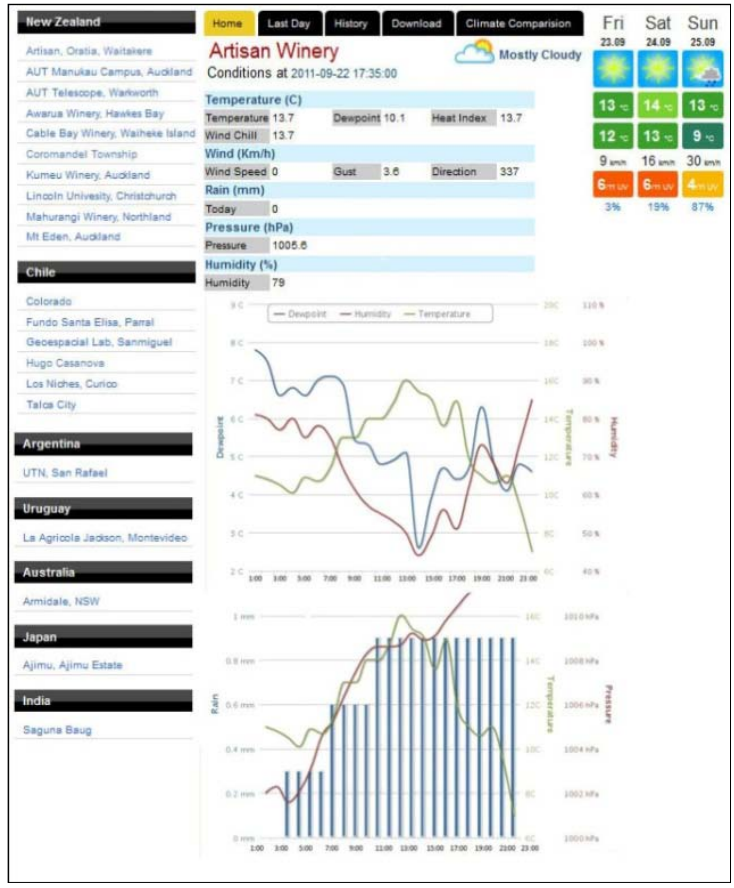

Figure 5: web monitoring of Artisan vineyard's environmental data (www.envirotelemetrics.com) 
Ghobakhlou et al., Development of Environmental Monitoring System with Wireless Sensor Networks

herein offer a range of benefits including energy conservation, hardware reuse and real-time performance.

Power and resource management are two main issues that needed to be taken into consideration. In this implementation, one way to achieve this is to incorporate a low level controller that facilitates for more sensors and computational power. The higher level controller will handle the ZigBee mesh networking, and request the data from the low level controller. The main task for the low level controller is to collect data from the sensors and process the data in suitable format and send it the high level controller on demand.

\section{CONCLUSIONS AND FUTURE WORK}

The paper investigated the recent advances in remote wireless sensor devices, and how WSN of these devices could be combined with the internet and used in vineyard operations, such as management decision making, by monitoring environmental conditions. The proposed system architecture was developed and prototype hardware was built. The baseline of the proposed system architecture was implemented with the capability of monitoring environmental data from twenty five base stations in five countries. Power management need to be improved by introducing more intelligent and efficient algorithms to reduce unnecessary up-time and redundant data transmissions.

Future work will employ more field installation of nodes with powerful base station to provide robust longterm monitoring of vineyards conditions. The network data security will also be taken into account in our future work.

\section{REFERENCES}

Camilli, A., Cugnasca, C.E., A. Saraiva, M, Hira-kawa, A.R. and Corrêa, P. L. (2007). From wireless sensors to field mapping Anatomy of an application for precision agriculture. Computers and Electronics in Agri-culture, 58(1),pp 25-36.

Cao, X., Chen, J., Zhang Y. and Sun,Y. (2008). Development of an integrated wireless sensor network micro-environmental monitoring system. ISA Transactions, 47(3), pp247-255.

Deborah, E., Ramesh,G., John, H. and Satish, K.(1999) . Next century challenges: scalable coordination in sensor networks. In Proceedings of the 5th annual ACM/IEEE international conference on Mobile computing and networking Seattle, Washington, United States: ACM Press, pp. 263-270.

Egan, D. (2005). The emergence of ZigBee in building automation and industrial control. Computing \& Control Engineering, 16(2), pp. 14-19.

Garcia, R.R. (2006). Understanding the Zigbee stack, Freescale Semiconductor Inc., pp. 1-2.

Ghobakhlou, A., Perera, A., Sallis, P. and Zandi, S. (2010), Modular sensor nodes for environmental data monitoring. Proceedings of the Fourth International Conference on Sensing Technology, University of Salento, Lecce, Italy June 3 5, 2010, pp 372-377 ISBN 978-0-473-16942-8

Ghobakhlou, A., Shanmuganthan, S. and Sallis, P. (2009). Wireless sensor networks for climate data management systems. In B. Anderssen et al. (eds) 18th IMACS World Congress - MODSIM09 International Congress on Modelling and Simulation, Australia, pp. 959-965.

Hirafuji, M., Kubota, T. ( 1994). Chaos of Plant Growth under Changing Environment. Environ. Control Biol. 32 (1), 31-39.

Jose D. and Gutierrez A. (2005). IEE Std. 820.15.4 Enabling Pervasive Wireless Sensor Networks. Innovation Centre, pp.254.

Laurenson, M.R., Kiura, T., Ninomiya, S. (2002). Providing agricultural models with mediated access to heterogeneous weather databases. Appl. Eng. Agric. 18, 617-625.

Lee, W.S. , Alchanatis, V. , Yang, C. , Hirafuji, M. and Moshou, D. , Li, C. (2010). Sensing technologies for precision specialty crop production. Computers and Electronics in Agriculture, Vol. 74, No. 1. pp. 2-33.

Portilla, J., De Castro, A., De La Torre, E. and Riesgo, T. (2006). A Modular Architecture for Nodes in Wireless Sensor Networks, Universal Computer Science (j-jucs), 12(3), pp 328-339.

Sallis, P., Shanmuganathan, S., Pavesi, L. and Jarur, M. (2008), A system architecture for collaborative environmental modelling research. in Waleed W. Samari and William McQuay (eds), International Symposium on Collaborative Technologies and Systems (CTS), IEEE, California, pp 39-47.

Sallis, P., Jarur, M., Trujillo, M., and Ghobakhlou, A. (2009). Frost prediction using a combinational model of supervised and unsupervised neural networks for crop management in vineyards. In B. Anderssen et al. (eds) /18th IMACS World Congress - MODSIM09 International Congress on Modelling and Simulation/, 13-17 July 2009, Cairns, Australia. ISBN: 978-0-9758400-7-8. pp. 789-795.

Srivastava, N. (2010). Challenges of next-generation wireless. Journal of Telecommunications, 1 (1).

Sohraby, K., Minoli, D. and Znati, T. (2007). "Wireless Sensor Networks: Technology, Protocols, and Applications". WileyInterscience

Suri, A., Iyengar, S. and Cho, E. (2006). Ecoinformatics using wireless sensor networks: An Overview. Ecological Informatics, 1(3), pp 287-293.

Wheeler, A. ZigBee Wireless Networks for Industrial Systems - White Paper, http://www.microcontroller.com/Embedded.asp?did=149, visited on 10/07/2011.

Yick, J., Mukherjee, B. and Ghosal, D. (2008). , Wireless Sensor Network Survey. Science Direct Computer Networks, Vol. 52, Issue 12, pp.2292-2330. 